\title{
NOTAS DE UNA “RECIENVENIDA” PARA PENSAR LA INVESTIGACIÓN EN CIENCIA POLÍTICA
}

\author{
Corina Echavarría*
}

\begin{abstract}
Hemos pasado de celebrar la participación ciudadana en los estudios de caso, a la necesidad de una profundización de los esquemas de comprensión de los procesos de ampliación de la democracia, que permitan el análisis comparado y la socialización de aprendizajes ciudadanos e institucionales. En este marco, el papel del profesional observador y analista entra en tensión en tanto realiza acciones de reconocimiento al nombrar el mundo desde la ciencia. En este trabajo partimos de la figura del "recienvenido" - M. Fernández -, para poner en cuestión la situación extranjería del investigador para la comprensión y estudio comparativo de las prácticas situadas, en una doble dimensión: la legitimidad de los saberes que se encuentran en los procesos de entendimiento que atraviesan la investigación y la inserción de la otredad del investigador en los procesos de autocomprensión de los colectivos ciudadanos.

Palabras Clave: Participación ciudadana. Método comparativo. Comprensión. Dialogo de saberes. Investigación acción.
\end{abstract}

"El nacer sólo una vez, aunque a nadie le está de más, y dura y no se olvida en toda la existencia, no rige para las ideas,

que viven de rejuvenecimientos no de continuidad"

(M. Fernández, 1923)

Después de casi treinta años de iniciada la redemocratización en América Latina, hemos pasado de celebrar la participación ciudadana en los estudios de caso, a la necesidad de una profundización de los esquemas de comprensión de los procesos de ampliación de la democracia (Echavarría, 2008a, 2008b). Parafraseando a Bobbio, ya no se trata de ver en cuántos lugares votamos los ciudadanos, sino de analizar cómo participamos; las condiciones, alcances y límites de los procesos participativos que se difunden e institucionalizan, especialmente, en el ámbito local. En tal sentido, mi preocupación se orienta hacia propuestas de investigación que permitan el análisis comparado y la socialización de aprendizajes ciudadanos e institucionales.

* Doutora em Administração. Pesquisadora do Conselho Nacional de Pesquisas Científicas e Técnicas (CONICET), Centro de Pesquisa e Estudos sobre Cultura e Sociedade (CIECS), da Universidade Nacional de Córdoba.

Duarte Quiros 96, 6B. Cep: 5000. Córdoba - Argentina. coriecha@hotmail.com
Las opciones por estrategias comparativas están vinculadas a la posibilidad de "superar" los límites del parroquialismo asociado a los estudios de caso, ampliamente utilizados en este campo de estudio. Por considerar que, si bien en los mismos se estaría dando cuenta de los fenómenos con gran "intensidad", las construcciones conceptuales y teóricas parecen ser de corto alcance y fuertemente determinadas por el contexto. No obstante, es importante señalar que la pretendida alternativa del método comparativo tampoco está exenta de limitaciones. Particularmente, en lo que refiere a la mencionada formación y construcción de conceptos, entre los que podemos destacar la clasificación equivocada de los casos en estructuras analíticas alienadas, la arbitrariedad en los recortes del fenómeno propiamente dicho - en pos de la comparabilidad por semejanza - y la ambigüedad conceptual que resulta de la extensión de las dimensiones de análisis en el esfuerzo por captar la diversidad emergente de los casos (Sartori-Morlino, 1994).

De lo que se trataría en este trabajo, entonces, es de considerar un giro metodológico cuidadoso para fundamentar la construcción 
conceptual en las experiencias políticas de ciudadanos y administraciones, involucrados en la implementación de instituciones de gestión participativa, así como para avanzar en la interpretación de los resultados de las observaciones particularmente situadas - por analogía, similitud o contraste -, señalando y poniendo en valor lo que dichas experiencias traen de nuevo o incorporan creativamente a la tradición democrática. Parafraseando a Lazamares (1990), podemos decir que buscamos hacer confluir las dimensiones explicativas e interpretativas en el estudio de los procesos participativos, trazando vínculos generales entre los distintos fenómenos, a partir del análisis de casos que, en su explicitación, identifiquen las aristas que los hacen singulares y den inteligiblidad a los caracteres que resultan de interés para la transformación democrática - el interés que orienta nuestra indagación.

No obstante lo dicho, coincido con los planteos realizados en debates actuales que afirman que la opción por el método comparativo, más allá de sus discutidas contribuciones a la construcción teórica, no resuelve los diversos problemas metodológicos presentes en la investigación cualitativa (estudios de caso) en ciencia política. Su consideración ofrece la oportunidad para iniciar un camino reflexivo ¥ en torno del método, de las técnicas de obserثิ vación y, particularmente, de la posición del Ф் investigador. En el último caso, de mi preo这 cupación, me refiero al papel del profesional is observador y analista que entra en tensión con los fenómenos y sus protagonistas, en tanto realiza acciones de reconocimiento al nombrar el mundo desde la ciencia.

Para tal fin, la estrategia analítica que se despliega en este trabajo busca alcanzar la comprensión y el estudio comparativo de las prácticas situadas, en una doble dimensión:

- La inserción de la otredad del investigador en los procesos de autocomprensión de los colectivos ciudadanos, para lo cual nos servimos de las dimensiones de análisis que abre la figura literaria del "Recienveni- do" de Macedonio Fernández, ${ }^{1}$ como punto de partida para nuestras reflexiones y para atravesar las discusiones desarrolladas por Simmel, Schutz, Elias y Habermas. ${ }^{2}$ Considerando que lo que Fernández enuncia en términos de "invisibilidad", cuando se refiere a la relevancia de los temas o problemas que puede abordar el Recienvenido en sus alocuciones, o al intentar interactuar con los miembros de la sociedad porteña, forma parte de las preocupaciones de los mencionados autores, al pensar la posición del investigador-extranjero.

- La legitimidad de los saberes que se encuentran en los procesos de entendimiento que atraviesan la investigación; en este caso, orienta nuestra reflexión la interrogación por el lugar la intersubjetividad, de la posibilidad del intercambio dialógico en la construcción de conocimiento, esto es, cuando los modos de conocimiento privilegian formas de validación exógena o "círculos de seguridad" e intereses que exceden y hasta son indiferentes con aquellos que se desarrollan en los contextos situados, tales como los escenarios de la participación. Para lo cual hacemos pie en los postulados de la "investigación-acción" inspirados en la pedagogía de Paulo Freire.

En tal sentido y retomando las observaciones iniciales al método comparativo, nos preguntamos: ¿Cómo integrarnos y hacernos visibles en los procesos de transformación de-

${ }^{1}$ Macedonio Fernández (1874-1952) era, en las palabras que Borges (1998) escribió para prologar la publicación de sus Obras Completas, un hombre al que "La erudición le parecía una cosa vana, un modo aparatoso de no pensar" y que "Vivía (más que ninguna otra persona que he conocido) para pensar". Si bien invitamos a recorrer las anécdotas y reflexiones acerca del personaje y amigo realizadas por Borges en el mencionado prólogo, a modo de síntesis presentadora, quisiéramos compartir su párrafo final: "Más allá de su doctrina filosófica y de sus frecuentes y delicadas observaciones estéticas, Macedonio nos ofrecía, y sigue ofreciéndonos, el espectáculo incomparable de un hombre que, indiferente a las vicisitudes de la fama, vivía en la pasión y en la meditación. No sé qué afinidades o divergencias nos revelaría el cotejo de la filosofía de Macedonio con la de Schopenhauer o la de Hume; bástenos saber que, en Buenos Aires, hacia mil novecientosveintitantos, un hombre repensó y descubrió ciertas cosas eternas.”

${ }^{2}$ Retomamos, con este propósito, los trabajos de George Simmel, "Digressions sur l'étranger" [1908]; Alfred Schütz, "El Forastero" [1944], Norbert Elias, "The established and the outsiders" [1965] y "Compromiso y distanciamiento" [1983] y Jürgen Habermas, "Teoría y Praxis” [1971]. En este último caso, con referencia en su segunda edición, porque en la Introducción a la Nueva Edición, el autor da cuenta de sus avances en el tema en Conocimiento e Interés, Estudios previos y complementarios a la Teoría de la Acción Comunicativa, entre otros. 
mocrática que investigamos? ¿Cómo construir la legitimidad social del proceso y el producto de las investigaciones, de manera de contribuir no sólo a la comprensión y comparabilidad de los casos, sino, fundamentalmente, a la socialización los aprendizajes que en ellos se realizan?

\section{EL RECIENVENIDO}

A seguir, entonces, propongo partir de la figura del "Recienvenido" - creada por el escritor argentino Macedonio Fernández -, con el objetivo de problematizar la situación de extranjería del investigador. En este ejercicio, podríamos caer en la tentación de referirnos al Recienvenido simplemente como "[...] aquella diferente persona notada enseguida por todos, que llegado recién a un país de la clase de los diferentes, tiene el aire digno de un hombre que no sabe si se ha puesto los pantalones al revés, o el sombrero derecho en la cabeza izquierda [...]" (Fernández, 1996, p. 31). Sin embargo, prefiero remitirme al fragmento, una nota a pie de página, que efectivamente me llevó a pensar en mi condición de investigadora. Es que, curiosamente, como nota cerrando el primer apartado en "Papeles de Recienvenido", Fernández ([1923] 1996, p. 15) sitúa su relato $\mathrm{y}$, de esa manera, nos permite empezar a conocer al Recienvenido:

Escrito en una Aldea donde la recienvenidez, de solo una vez, no se le saca a uno nunca. En Buenos Aires, que estima inverosímil haber vivido hasta los treinta a cuarenta sin conocerla, por lo que hay que sacarse pronto la recienvenidez tardía, todo el primera vez llegado, que conoce en los semblantes el mal gusto del no haber nacido en ella, se apresura a dar una instruidísima conferencia sobre "La Argentina y los argentinos" tres días después de haber desembarcado. Esto da resultado; se comprende que conferencia tan pronta y con tal tema no es la colosal fatuidad y entrometimiento ignorante que suele sospecharse, sino la ansiedad por quitarse cuanto antes la pátina de recienvenidez. Ser "recienvenido" en Buenos Aires ni por un momento se perdona; es como insolencia (Destacado de la autora).
Entonces, podemos comenzar por decir que la "recienvenidez" se trata de una condición - dificultosa de superar - propia del que recién viene, lo cual no aclara mucho. Pero, para avanzar, debemos notar que se trata de una cualidad que parte de la percepción del otro. No ha "llegado" recientemente, sino que "vino" donde otros ya estaban. En tal sentido, la recienvenidez puede ser reconocida en la mirada de los "otros" que integran el colectivo al cual el recienvenido se integra.

Se trata de una condición de la que no se exceptúa a nadie, no obstante lo cual resulta inverosímil y hasta un descaro o atrevimiento para el colectivo de referencia. Por tal motivo, pareciera imponerle al Recienvenido, como necesidad, la urgencia de dar cuenta de un saber (¿compartido?) referido al mismo, mediante el uso de la palabra. En esta necesidad de hablar, por no estar callado, advierte el autor, apresurado habla idiomas que no sabe, sin establecer necesariamente un diálogo.

No obstante lo cual podríamos asumir que, en busca del propio reconocimiento, dicho monólogo "erudito" tendría, como horizonte, no la mera exposición de su saber, sino la aceptación del público referido, por lo cual lo estaría incluyendo deliberativamente. También en el relato de sus experiencias, el Recienvenido se refiere a los esfuerzos que realizaba por establecer un "piso" común para el diálogo con los otros: "[...] la tarea de bajarme las rodillas para no quedarme en el aire me imposibilitaba toda atención y cortesía” (Fernández, 1996, p. 34). Un contexto de significación que condiciona la fluidez del intercambio intersubjetivo y le demanda un esfuerzo especial de horizontalización. Esto es, el Recienvenido, además de reconocer el carácter alienado de sus marcos de significación, debemos atender, atribuye una posición de superioridad al propio saber.

Avanzando en los "papeles" con el foco puesto en nuestras reflexiones, podemos advertir que el Recienvenido se autodefine a partir de diferentes cualidades, las cuales resultan de su condición y de sus competencias respec- 
to del grupo al que se incorpora. Entre ellas, destacamos: "sintético" y "evidente". El Recienvenido puede reparar en las "pequeñeces" que, según el autor, los otros, por el "alumbramiento” de la pertenencia, desestiman. Así, en clara analogía de la observación con la lectura, busca los "agujeros" que, en la comprensión, le genera la palabra de los otros: "En cuanto al agujero que yo buscaba era uno que me había hecho en la mente una reciente lectura [...]" (Fernández, 1996, p. 26).

Sin embargo, somos advertidos también acerca de los relativos alcances de su pretendida certeza y claridad en la observación. El Recienvenido puede resultar "invisible" al grupo por la irrelevancia de los problemas que lo llevan al lugar: "[...] se concentra en una meditación sobre eclipses, ceguera de los transeúntes, huelga de los repartidores de luz, invisibilidad de los átomos y del dinero de papá, y así logra no ser visto" (Fernández, 1996, p. 31-32).

Finalmente, encontramos que, cuando se "confiesa", el Recienvenido nos remite al estado de confusión que caracteriza a la persona que "por primera vez es un recién llegado". Obsérvese que cambia la perspectiva. Entonces, en un ejercicio autoreflexivo - ya sin tomar como centro las percepciones de los otros -, da cuenta de sus propios límites en las

* lecturas que realiza de la Aldea - "esforzados ¿ estudios y brillantes primeras equivocaciones"

Ф் - y, además, admite que "no se decide a cercio迹 rarse del desperfecto en público" con los otros is (Fernández, 1996, p. 31).

\section{PROBLEMATIZANDO LAS FORMAS DE HACER INVESTIGACIÓN}

Notas sobre la alteridad del investigador

Como decíamos, nos servimos como punto de partida de la figura literaria del Recienvenido para actualizar reflexiones sobre la producción de conocimiento en las ciencias sociales, que atravesaron el Siglo XX y que, consideramos, mantienen total vigencia cuando se trata de pensar el estudio de procesos participativos de manera comparativa (y aún en formato de caso). Particularmente, si tenemos en cuenta que, como decíamos al introducir el trabajo, de lo que se trata es de comprender experiencias situadas que nos permitan contribuir a la transformación democrática de los procesos de toma de decisiones públicas.

A principios del siglo pasado, Simmel apelaba a la metáfora del extranjero para ahondar, en sus reflexiones, sobre el oficio de la sociología y, particularmente, para referirse al esfuerzo de objetivación. Casi veinte años más tarde, Schütz (1999), en un ensayo de psicología social, se propuso estudiar la "situación de acercamiento" en la que se encuentra un forastero cuando quiere interpretar el esquema cultural de un grupo social al que se incorpora, no sin hacer también ciertos paralelismos con el oficio del sociólogo. Finalmente, en la segunda mitad del siglo, Elias reflexiona sobre la construcción social del "extraño" y del conocimiento en ciencias sociales, mientras Habermas enfoca, en la historización de la "situación de intereses" de la que participa, el acto de conocimiento y la "peculiar condición" del sujeto de conocimiento. Todos ellos, de diferente manera, convergen y aportan elementos para la reflexión en torno de la posición y prácticas del investigador respecto de los fenómenos y sujetos que observa y busca comprender.

\section{Recién-llegado al país de los dife- rentes}

En términos generales, podemos comenzar por afirmar que las metáforas del extranjero, del forastero, del extraño, entre otras, hacen referencia a aquel que llegó y que ha de permanecer. No se trata de un nómada, ni de un sedentario.

El extranjero de Simmel se define, entre otras características, por su ausencia originaria. Desde el punto de vista del grupo, coincide 
Schütz, es un hombre sin historia. Si bien está vinculado al grupo, no pertenece al mismo desde el origen.

Se distingue, entonces, de los miembros incondicional y completamente identificados con el grupo. Porque, además de no pertenecer desde siempre al espacio social en cuestión, el extranjero de Simmel y Schütz incorpora "[...] cualidades que presuntamente no proceden ni pueden proceder de él [...]", porque "encuentra", en los esquemas de referencia que trae consigo, una pauta que considera válida para el grupo y que, "inevitablemente", le resultará inadecuada (Santamaría, 1994, p. 65). Como afirma Santamaría (1994, p. 65), parafraseando a Simmel, es un “[...] intruso, que con diferentes expresiones sociales habita en esa tensión del afuera [exterioridad] y enfrente [oposición] pero dentro [posición interna y pertenencia] [...]".

Se trata de una alteridad que se fija, que se implanta en el grupo provisional, aunque duraderamente, porque "aunque se haya detenido, no se ha asentado completamente" (Simmel apud Santamaría, 1994, p. 65). Como ha de quedarse, se diferencia también del viajante exterior y sin compromiso - nómada. Porque, al establecerse, parafraseando a Santamaria (1994), demanda la creación de situaciones de interacción, al tiempo que se configura en el entramado del grupo.

Esto da cuenta de que la figura del outsider no es de carácter ontológico, sino relacional: es en la relación que la idea del otro adquiere sentido. Está sujeta a una comunidad que la configura y la construye como tal y tiene un carácter predominantemente "abstracto”. Dado que, como señala Simmel (apud Santamaría, 1994, p. 66), “[...] con el extranjero solo se tiene de común ciertas cualidades de orden general [...]", la relación “[...] no es el resultado de la existencia de características compartidas y diferenciales entre los individuos, sino que tiene su origen [...] en el carácter genérico, vago, en que los miembros sienten esa comunidad". ${ }^{3}$

${ }^{3}$ Cabe destacar que los recién llegados son extraños no

\section{Construir espejos o leer los agujeros}

¿Cómo interpreta el forastero el esquema cultural del grupo social al cual se acerca? ¿Cómo se orienta dentro del grupo? Podríamos decir que estas son las preguntas que permiten a Schütz hablar de lo que denomina la "situación de acercamiento".

En este caso, adquiere relevancia, nuevamente, la distinción entre el extranjero y los miembros plenamente identificados o situados en el grupo, entre el observador y el actor. El "actor situado" es aquel para quien el mundo social es el campo de sus actos y, secundariamente, "objeto de su pensamiento" o de conocimiento. Este conocimiento, consecuentemente, se organiza en términos de la significatividad para dichas acciones. La disponibilidad técnica y la comprensibilidad intersubjetiva son los intereses que guían el conocimiento cotidiano y que hacen accesible la experiencia, son sus condiciones de posibilidad. Este conocimiento, además, adquiere para el actor situado la "[...] apariencia de una coherencia, claridad y congruencia suficientes como para ofrecer a cualquiera una probabilidad razonable de comprender y ser comprendido" (Schütz, 1999).

Como señalábamos anteriormente, en principio, el extranjero no comparte esa "pauta cultural de la vida grupal", o los supuestos básicos del orden simbólico materializados en el sistema social que viabilizan la intersubjetividad y, por tanto, interpreta el nuevo ambiente -en tanto campo de aventura - a partir de su "pensar habitual". Pasa a ser, según Schütz (1999), “[...] el hombre que debe cuestionar casi todo lo que le parece incuestionable a los miembros del grupo". Sin embargo, esta actitud de "observador", sobre lo que vive como problemático y difícil de solucionar - la pauta cultural -, mediante la cual objetiva a los sólo en relación con los "viejos residentes" o miembros del grupo originario, sino también entre sí. Ver el trabajo de Alcalde Campos (2011), donde la autora reflexiona en torno de la obra "The stablished and the outsiders" de Elias y Scotson. 
miembros del grupo en la interpretación, genera un tipo de conocimiento "aislado". Un conocimiento de efecto "especular" que, prescindiendo de la intersubjetividad, resulta insensible e irresponsable y susceptible de sumirse en prejuicios, parcialidades y malentendidos.

La metáfora del espejo vuelve con Elias (1990, p. 26), esta vez como una necesidad de los grupos. Según el autor, es necesario colocar frente a los grupos "[...] un espejo en el que puedan verse a sí mismos tal como los vería no un crítico comprometido del grupo antagonista, sino un observador distanciado [...]", para que los grupos puedan "distinguir las realidades de las fantasías" en la amalgama de observaciones realistas y fantasías colectivas que generan.

El distanciamiento u "objetividad" es una de las características básicas comúnmente reconocida por los autores al extranjero. Actitud que, según Schütz (1999), en el forastero, deviene de "su necesidad de adquirir un pleno conocimiento de los elementos de la pauta cultural abordada, y de examinar con cuidado y precisión, con este fin, lo que el endogrupo considera que se explica por sí solo”. Busca los "agujeros de sentido" en el orden al que desea acceder. Mientras que, en el caso del sociólogo, particularmente, lo vincula al hecho de que se abstiene intencionalmente de participar de * la red de planes, instituciones y sistemas de จे orientación de los actores. La objetividad es, ®ึ aquí, sinónimo de desinterés. ลิ investigador vinculación e interés por el grupo, $\therefore$ al mismo tiempo que distancia de sus "particularismos" y (o) parcialidades. Para el autor, ese esfuerzo no puede ser entendido como desinterés (o la pretendida neutralidad), ni como la ausencia de participación (observación teórica), sino como una "combinación particular entre la proximidad y la distancia, la atención y la indi- ferencia” (apud Pires, 2008, p. 83).

En tal sentido, debemos considerar que se altera la naturaleza del espejo al que hacía referencia Elias (1990, p. 11), ya que, para el autor, “[...] no se puede afirmar en un sentido absoluto que la actitud de una persona sea distanciada o comprometida [...]”, y, fundamentalmente, que la condición de posibilidad de la comprensión de los problemas sociales es la participación y el compromiso personal de los investigadores.

Así mismo, Habermas (2000) destaca la importancia de tomar en cuenta las relaciones de intersubjetividad, para lo cual pone de relieve, en el marco de la construcción de un conocimiento crítico, la relación de participación del sujeto que comprende con respecto a otro que está frente a él, antes de garantizar el anonimato e intercambiabilidad del que observa. Porque el acercamiento del investigador, desde esta perspectiva, es una interrogación, una comunicación que, para poder encontrar al otro que está frente a él, a nivel de la intersubjetividad de un entendimiento posible, no se puede ser indiferente a lo específico de la comunicación cotidiana y sus bloqueamientos y obstáculos. Dirá Villasante (2004): "su realismo mágico [de la ciencia] exige partir de la realidad vivida"

\section{Libertad para viajar}

El extranjero, como se ha podido ver, no abandona su libertad de desplazarse, inclusive pensando en aquel que es miembro del grupo desde el principio (estado-observador-del-sistema o participante-crítico). Lo importante es que, viniendo de fuera o del interior del grupo, siempre se haya atribuido la libertad de viajar, que aprendiera a tomar alguna distancia respecto del grupo, que conservara su "elemento frio". Se trata de una triple libertad de desplazamiento (Pires, 2008): de conocimiento, política y ética.

- La libertad de conocimiento (a la que hacíamos referencia en el apartado anterior), es 
aquella que le permite introducir diversas formas de demarcación y poner en cuestión los consensos del grupo. El extranjero no está en el grupo simplemente para asentir, sino que su función, para la autoconciencia del grupo, acerca de lo que condiciona la acción y la aprehensión del mundo, es indispensable. Sin embargo, esta libertad encuentra límites, porque, como advierte Schütz (1999), la condición de atopos del forastero, de persona libre, también representa una amenaza para la cohesión social del grupo:

[...] en todos estos grupos existe un grado de distanciamiento que ninguno de sus miembros puede sobrepasar sin aparecer a los ojos del grupo como hereje (y convertirse en tal), sin importar que sus ideas y teorías concuerden con los hechos observables y se acerquen a aquello que llamamos 'verdad' (Elias, 1990, p. 26).

- La libertad política los faculta a desplazarse, a ir y venir en los posicionamientos del grupo de referencia. En términos políticos, se constituye como atopos, síntesis de la lejanía y proximidad a la que hemos hecho referencia, una "figura social objetiva" o desinteresada respecto de las pautas del grupo, aunque no sin intereses. Según Simmel, no está “[...] constreñid[o] por intereses de grupo y de fracción, que no está ligad[o] por ninguna consideración que pudiera constituir un prejuicio para la percepción, la comprensión y estimación justa de los hechos y decires". (Santamaria, 1994, p. 66)

En este contexto, el científico crítico no sólo da cabida a la autocomprensión metodológica del "interés" que está en la raíz del conocimiento, vinculando el contexto de surgimiento y utilización de los saberes del grupo, sino que se caracteriza por hacer lugar al interés emancipatorio que guía el conocimiento (por encima del cognoscitivo práctico y técnico al que aludimos anteriormente). Esto es, el interés por la liberación en el conocimiento, por la praxis política que aspira a subvertir el sistema de dominación (Habermas, 2000).

- La libertad ética de tener en cuenta otros grupos subalternos. Las reflexiones en torno de los extranjeros trabajan también la analogía del extraño con los pobres y los "otros", o enemigos internos del grupo, esto es, aquellos cuya identidad no confluye totalmente con el grupo y (o) no le son incondicionales. Además, señalan que los forasteros no son extraños solamente para los actores situados, sino también para estos "otros" dentro del grupo. En este contexto, el distanciamiento de los científicos sociales, que "[...] no pueden evitar experimentar esas interrelaciones, directamente o por identificación, como participantes inmediatos en estas [...]", se pone en juego en la comprensión y en "[...] hacer que los demás comprendan, cambiantes conjuntos de interrelaciones formadas por los seres humanos, la naturaleza de esos lazos y la estructura de esos cambios" (Elias, 1990, p. 23).

\section{Notas sobre los saberes legítimos}

La problematización de la relevancia social del conocimiento, que se produce en el contexto de los procesos de la investigación académica, fue hasta aquí enunciada en términos de riesgos del aislamiento del saber que se genera en el acercamiento a los grupos sociales. También, a través del Recienvenido y de las prácticas de los diferentes "extraños", se explicitan las dificultades para poner en diálogo su "pensar habitual" con los saberes que sustentan las prácticas e intercambios cotidianos en los grupos involucrados en los procesos de investigación.

Las propuestas de "flexibilización" de las formas de investigar y la consideración de la circulación de saberes que acontecen en dichos procesos ha sido ampliamente problematizada - desde la década de setenta - por las diversas propuestas de investigación-acción. En el desafío de abandonar los "círculos de seguridad" (Freire, 2002, p. 26), que ofrecen las metodologías de la ciencia tradicional, se ha avanzado en la recuperación de la historicidad y la intersubjetividad en los procesos de indagación, así como de la relevancia social tanto del proceso como de los resultados para los grupos con los cuales trabajamos (Wigdor; 
Barrionuevo; Echavarría, 2012).

Así, con base en el interés emancipatorio del conocimiento, autores como Freire (2002) ya no se refieren al extraño, sino al "Hombre Radical”. La mirada está en la actitud del investigador, del que recién llega, que se inserta en la realidad del grupo sin "temer" al diálogo con los otros. Considerando que, mediante la interacción comunicativa, estos otros actores conocen lo que es diferente a sí mismos, reconocen al otro y se reconocen a sí mismos en el otro, se encuentran, todo lo cual contribuye, según el autor, a la construcción de un saber “cada vez mayor" para ambos.

En este contexto, el recién-llegado, con su sola llegada, es capaz de realizar un acto de ruptura con la red de relaciones y referencias ya existente, porque su acción no tiene, no puede tener lugar en el aislamiento. Su iniciativa cambia el mundo, un mundo que comparte con otros, que estaba antes y que continúa después, al tiempo que revela elementos sobre sí mismo. Desde esta perspectiva Arendt (1997, p. 77) nos permite pensar, entonces, en la "natalidad" del extranjero, y no en la potencial amenaza que la sola presencia del investigador podría representar para la continuidad del grupo con el que se relaciona en la construcción de conocimiento. Pensamos, con la * autora, que su llegada inicia una cadena por la ㄱ que el mundo, la realidad del grupo con el que ڤ્ป se interrelaciona, “[...] a través de la espon-

\& como la "rebelión de los laboratorios". El autor ลิ trataba de advertir y dar cuenta, con esto, sobre $\therefore$ la forma en que los sujetos con los que interactuamos en nuestras investigaciones nos inter艿 pelan, cuestionan nuestras formas de conocer e, inclusive, aportan técnicas, metodologías y posicionamientos para la producción de conocimiento científico. Los “actores situados”, que participan de nuestras investigaciones o apro- ximaciones, no sólo aportan sus "asombros e intuiciones”, sino que nos interpelan desde sus prácticas y experiencias.

De las experiencias previas de investigación-acción realizadas en espacios participativos, a nivel local, tanto en Brasil como en Argentina, ${ }^{4}$ he advertido necesario pensar en la posición que ocupa el investigador para el abordaje del proceso de construcción de conocimiento, los prejuicios relacionados a los diversos casos bajo estudio, tanto como la situación de poder en cada uno. Es decir, se plantea como una necesidad, en el devenir de la experiencia, el problematizar las relaciones de poder, las situaciones de intercambio con los actores involucrados en cada uno de los procesos, especialmente con aquellos directamente vinculados a la administración pública. ${ }^{5}$ Es por eso que, en línea con los planteos hasta aquí desarrollados, extraemos balizadores o bases que, para algunos de nosotros, son también "apuestas” para la revisión de las prácticas en términos dialógicos. ${ }^{6}$

\section{Situaciones concretas}

Enraizados en condiciones temporales y espaciales que los marcan, afirma Freire (2002,

${ }^{4}$ Nos referimos a proyectos de investigación asociados a la democratización de la gestión local que pusieron en foco los espacios participativos de toma de decisiones en ciudades de porte medio tales como Porto Alegre (Brasil) y Córdoba (Argentina), así como experiencias de extensión universitaria vinculadas a localidades menores, tales como municipios del interior del Estado de Bahía (Brasil) y de la Provincia de Córdoba (Argentina), y al desarrollo del Grupo de Trabajo Democracia Participativa de la Red Ciudadana Nuestra Córdoba.

${ }^{5}$ El contexto de producción de la investigación es diferente en Porto Alegre, donde la posición de "investigadora extranjera" modifica totalmente la disposición de todos los involucrados para el intercambio. El interés en hablar por parte de los funcionarios se vincula, entre otras cosas, a la necesidad de difusión de una experiencia innovadora que es llevada adelante por una fuerza de oposición en el Brasil, en tanto que en lo que se refiere al resto de los ciudadanos participantes consideran un "halago" la presencia de investigadores extranjeros, un "reconocimiento" del espacio logrado que contribuye a su reafirmación.

${ }^{6}$ Agradezco al equipo de la cátedra de Psicología Comunitaria de la Facultad de Psicología de la Universidad Nacional de Córdoba, por compartir conmigo sus documentos de trabajo, a partir de los cuales ordené las ideas que expongo a continuación. 
p. 130), los hombres "son porque están en situación”. La reflexión sobre esta situacionalidad equivale a pensar la propia condición de existir. De allí la importancia de partir de las prácticas y experiencias que acontecen en un espacio y tiempo determinado, tales como la implementación de instituciones de gestión participativa, como un punto de partida para pensar colectivamente la transformación, particularmente la democrática. Considerando que, en estas situaciones, los actores - históricos - se hacen y rehacen socialmente y desarrollan un "sentir común" de la realidad, la cual consideramos "[...] no puede ser vista, mecanicistamente, separada, simplistamente bien "comportada", sino en la complejidad de su permanente devenir.” (Freire, 2002, p. 130).

Por otro lado, es la propia situación, según los psicólogos comunitarios, la que motivaría el involucramiento y la movilización de los diferentes actores en la construcción de conocimiento. Como parte de los procesos participativos y por la experiencia desarrollada en ellos, los actores situados estarían motivados para actuar sobre sus problemas en la búsqueda de respuestas y soluciones referidos a los procesos de implementación de las instituciones. Esto es, trabajar con y sobre las contradicciones e inconsistencias que constituyen dichos espacios y que sus prácticas cotidianas asumen en ellos, es decir, en las palabras del Recienvenido: buscar conjuntamente los "agujeros".

Además, a diferencia del observador externo y "enjuiciador", la investigación situada incorpora como parámetro de relevancia y pertinencia el valor social de lo investigado y de sus resultados. El que sólo podría ser dimensionado, en su totalidad, en las situaciones concretas que comparten la problemática investigada con la constelación de condiciones y preocupaciones de los actores.

\section{Razonamiento público}

El diálogo, sostiene Freire (2002), es la herramienta que tenemos para no permanecer en el aislamiento, para no construir conceptos alienados y (o) invisibles; pero demanda una relación horizontal y democrática. Recuperar el intercambio argumentativo supone reconocer una diversidad de voces que sostienen diferentes maneras de ver la realidad, que realizan múltiples lecturas del mundo (saberes diferentes) que entran en diálogo.

En este sentido, remarca Villasante, metodológicamente, no basta con dar la palabra para romper las barreras del saber "establecido" y la cristalización de sus diferencias en una jerarquía que obstaculiza la comunicación. Es necesario reconocer que no es suficiente "lo que se dice”, sino que también intervienen en el encuentro las "expectativas y las ideas que cada quien tiene del otro que habla" y otros elementos paralingüísticos de la situación de habla que provienen de la trama de relaciones y sentidos en la que los actores están incluidos (AAVV, 2010, p. 5).

Además, pensar en un horizonte de entendimiento deliberativo trae consigo la dimensión radicalmente democrática del espacio público: la publificación irrestricta de asuntos. Esta se vincula con la posibilidad de discutir, objetivar, preguntar, interpelar, socializar, en síntesis, problematizar inclusive los modelos de indagación y sus resultados en los procesos de construcción de conocimiento. Lo cual no significa evitar la conceptualización o el razonamiento analítico, sino, precisamente, fundar la construcción de dichos conceptos, "nuevos conceptos", en los intercambios y consensos posibles de las situaciones concretas y sus tramas de sentido, considerando los límites contingentes de los mismos y las diferentes competencias comunicativas de los participantes. En tal sentido, se trata de una tarea de aprendizaje conjunto, en la que estamos todos implicados, aunque cada uno de los que participan "lo vivan de forma distinta” (Villasante, 2004, p. 5). 


\section{Intencionalidad de cambio}

Esta línea está generalmente relacionada, en el marco de las propuestas de investigación -acción, con la posibilidad de reflexionar críticamente sobre la realidad en la que estamos inmersos. Sin embargo, este trabajo refiere, particularmente, a la explicitación de los intereses que motivan y orientan la investigación.

En este sentido, hablamos de la dimensión política que tiene la "llegada" del investigador, un potencial transformador que se asocia no sólo a la novedad de su irrupción en un estado de cosas y relaciones pre-existentes, a lo que podría inclusive pensarse como "involuntario", sino fundamentalmente a los interrogantes que trae consigo. Destacando, que el investigador toma decisiones que no son asépticas, desde que selecciona los casos y construye las preguntas - monológica o dialógicamente -, estas están enmarcadas, al menos inicialmente, en su "pensar habitual" - una argamasa de su perspectiva disciplinar, marco teórico, posicionamiento ideológico e inquietudes.

Es por eso que, en este contexto, se enfatiza, también, la necesidad de explicitar incorporar a los contenidos del diálogo - las relaciones que el investigador construye a lo largo del proceso entre dicha argamasa y las F lecturas que realiza - aislada o colectivamente ऽ - de las prácticas cotidianas, saberes en acto y

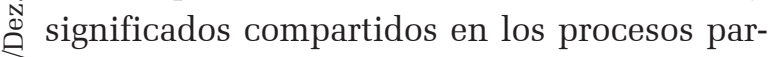
离 ticipativos.

\section{NOTAS FINALES}

“...con la más delicada cordialidad que una ciudad ima-

[...] se adelanta al recienvenir de todos dándole el sabor de un permanecer, y así ha hecho millones de permanecedores que venían con el algo despectivo 'de paso'.” (M. Fernández, 1923)

La experiencia de investigación basada en el estudio de diferentes casos y la comprensión de experiencias de ciudadanos y ciudadanas en la participación en diversos contextos instaló, en mí, como una necesidad, la inquietud de reflexionar en torno de la posición que ocupaba y ocupo cada vez que me acerco a ellos, los procesos de construcción de los lazos de confianza que nos permitieron avanzar en las entrevistas y las tensiones devenidas de iniciativas apresuradas, descontextualizadas o, al menos, insuficientemente explicadas para aquellos que compartían conmigo la construcción de conocimiento en cada investigación. Como señalé anteriormente, andando advertí que estas reflexiones no introducían, por sí, novedad en el debate pre-existente o en el mundo académico que era antes, antes de mis limitadas experiencias y las de muchos otros que, con los procesos de democratización participativa de las decisiones, a nivel local, empezábamos a circular compartiendo experiencias de acción-reflexión-acción en la definición de asuntos públicos.

Ciertamente, se trata de preocupaciones que se rejuvenecen resignificadas en los desafíos epistemológicos y metodológicos de nuestro campo de conocimiento: la ciencia política. Actualmente, estamos preocupados por la comparabilidad de lo que los estudios de caso apuntan como creación para la ampliación democrática en contextos particulares y por la posibilidad, en orden a un interés emancipador, de la socialización de aprendizajes no sólo institucionales sino también de los actores involucrados en dichos procesos. En este trabajo, hemos puesto el eje de nuestra exposición en dos cuestiones: la otredad - investigador -, que nombra el mundo, y los saberes que confluyen en la definición de esta acción.

Podemos decir que todo proceso de investigación inicia una cadena de relaciones con los grupos de referencia e irrumpe en un estado de cosas, en un entramado de relaciones y significados, a partir de lo cual se configura la posición del Recienvenido-investigador. Éste, del “afuera”, incorpora marcos de sentido que, aunque familiares para él, resultan ajenos a los miembros del grupo cuyas prácticas y experiencias se constituyen el foco del co- 
nocimiento colectivo, cuanto más si estamos pensando en la posibilidad de que distintos grupos participen simultáneamente del mismo proceso (inclusive a través de las fronteras nacionales). En tal sentido, nos advierte la figura creada por Macedonio Fernández, el peligro es la invisibilidad de las preguntas o de los problemas que, resultando de manera aislada de este pensar habitual del Recienvenido-investigador, aparecen irrelevantes respecto de las contradicciones, tensiones y (o) límites que experimentan los procesos participativos, cuando no lo posicionan "en frente", por la imagen deformada que devuelve el espejo que se ha creado para mirar las prácticas de los grupos o, inclusive, por la actitud que asume.

El desafío está en pensarse, en actuar "dentro", como afirmáramos desde la investigación-acción. Esto pone de relieve los saberes que circulan. En primer lugar, considerando que en términos abstractos, representamos para el grupo un saber legitimado -socialmente reconocido -, más allá de sus fronteras e independiente intruso de las relaciones que se establecen en el mismo. No obstante lo cual, compartimos con ellos la cualidad del interés por el tema en cuestión: la participación, la ampliación democrática. Esto nos da el primer "sabor" de permanecer y pertenecer, a partir de lo cual parece posible establecer una relación de carácter intersubjetivo y no meramente objetivante $\mathrm{y}$, consecuentemente, jerárquica.

Es por eso que, en segundo lugar, las condiciones de posibilidad del diálogo deberían ir más allá de los intereses prácticos, cognitivos o emancipatorios compartidos y remitirnos a pensar en la forma en que se cristalizan nuestras diferencias. Diversos saberes, que confluyen en las experiencias participativas e interactúan en el proceso de investigación, demandan un esfuerzo de horizontalización intencional de nuestra parte, que busque poner en cuestión y problematizar, o al menos, desde una perspectiva pesimista, 'poner entre paréntesis' las jerarquías en estos ámbitos de construcción de conocimiento. Y, en tal sen- tido, dar atención al vínculo de reciprocidad que supone entablar este diálogo horizontal y pretender interrogar los consensos del grupo.

Finalmente, así como la ciudad cuida al Recienvenido, de su "despectivo de paso", admitiendo su prematura permanencia, los recorridos de los diversos autores ponen atención a la pertenencia del Recienvenido-investigador, atribuyéndole desde la diferenciación, primero, el compromiso y atención en las situaciones concretas que buscan comprender, como condición de posibilidad, después, de la capacidad de objetivación y síntesis, que le permitirían dar cuenta de los agujeros de sentido del orden institucional y simbólico que guía las prácticas ciudadanas. Como hemos señalado a lo largo del trabajo, distanciamiento no es sinónimo de indiferencia o desinterés, sino trabajo a partir, pero no bajo, de las pautas e intereses del grupo, en una construcción colectiva de nuevos conceptos y significados que nos permitan poner en común lo que de particular desarrollan las experiencias.

Recebido para publicação em 10 de outubro de 2012 Aceito em 09 de março de 2013

\section{REFERENCIAS}

AA/VV. Investigación acción participativa. Documento de trabajo. Cátedra Psicología Comunitaria, Facultad de Psicología, UNC. Córdoba, 2010. (mimeo)

ARENDT, Hannah. ¿Qué es la política? Barcelona: Paidós, 1997.

BARD, Gabriela Wigdor; BARRIONUEVO, Laura; ECHAVARRIA, Corina. Lo natural es pensar diferente: reflexionando sobre las praxis emancipadoras en las prácticas de investigación. En: PEYLOUBET, Paula (coord.). Co-construcción interactoral del conocimiento. Buenos Aires: Ediciones Nobuko, MinCyT, CIECS, UNC, p. 63-84, 2012.

BIRULÉS, Fina. Introducción: ¿por qué debe haber alguien y no nadie? En: ARENDT, Hannah. ¿Qué es la política? Barcelona: Paidós, 1997.

BOBBIO, Norberto. El futuro de la democracia. Santafé de Bogotá: Fondo de Cultura Económica, 2000.

BORGES, Jorge Luis. Macedonio Fernández. En: Prólogos con un prólogo de prólogos. Buenos Aires, Alianza Editorial, 1998 [1975].

CAMPOS, Rosalina Alcalde. De los outsiders de Norbert Elias y de otros extraños en el campo de la sociología de las migraciones. En: Revista Papers, 96/2. p. 375-387, 2011. 
ECHAVARRÍA, Corina. Ciudadanos y procedimientos democráticos: sus tensiones y contradicciones. En: Revista Controversia, n. 191. Colombia, 2008a.

. De la cantidad a la calidad: pensar la participación después de 20 años. En: Revista Organizações e Sociedade, A Gestão do Futuro e o Futuro da Gestão: Desenvolvimento Social de Territórios, v. 15, n. 45, abr./jun. 2008, ISSN 1413-585x. Salvador: UFBA, 2008b.

Comunicación, ciudadanía y transformación democrática. Segundas jornadas de ciencia política: los desafíos de la Ciencia Política de cara al Bicentenario. Universidad Nacional de Rio Cuarto. Río Cuarto, 2008c.

Dialogar y participar: algunos apuntes a partir de la experiencia cordobesa. III Jornada de Investigación, Facultad de Ciencia Política y Relaciones Internacionales, UCC. Córdoba, 2010. (mimeo)

ELIAS, Norbert. Compromiso y distanciamiento. Ensayos de sociología del conocimiento. Barcelona: Ediciones península, 1990.

FERNANDEZ, Macedonio. Papeles de recienvenido y continuación de la nada. Obras Completas - Tomo IV. Buenos Aires, Argentina: Corregidor, 1996.

FREIRE, Paulo. Pedagogía del oprimido. Buenos Aires: Siglo XXI Editores Argentina, 2002 [1970].

FREIRE, Paulo. Conscientização: teoria e prática da libertação: uma introdução ao pensamento de Paulo Freire. São Paulo: Centauro, 2005.

GUTIERREZ, Juan; DELGADO, Juan Manuel. Teoría de la observación. En: GUTIERREZ, J; DELGADO, J M. (Coords.) Métodos y técnicas cualitativas de investigación en ciencias sociales. Madrid: Editorial Síntesis, p 141-173, 1995.
HABERMAS, Jürgen. Introducción a la nueva edición: algunas dificultades en el intento de mediar teoría y praxis [1971]. En: Teoría y praxis: estudios de filosofía social. Madrid: Tecnos, p. 13-48, 2000

MORLINO, Leonardo; SARTORI, Giovanni. La comparación en las ciencias sociales. Madrid: Alianza, 1994.

NOHLEN, Dieter. Método Comparativo. Disponible en http://www.rzuser.uni-heidelberg.de/ k95/es/doc/ diccionario metodo-comparativo.pdf

PIRES, Alvaro. Sobre algumas questões epistemológicas de uma metodologia geral as ciências sociais. En: POUPAR, Jean (et all). A pesquisa cualitativa-enfoques epistemológicos e metodológicos. Petrópolis: Vozes, 2008.

SANTAMARÍA, Enrique. "Extranjero", nada menos que una palabra mayor. En: Revista Papers, 1994, 43. p. 63-70.

SCHUTZ, Alfred. El forastero. Ensayo de psicología social. En: Estudios sobre teoría social. Buenos Aires: Amorrortu Editores, 1999. Disponible en http://bibliotecadigital. academia.cl/handle/123456789/624

VALDUECO, Iván Lamazares. Método comparativo. En: Román Reyes (Dir.). Diccionario Crítico de Ciencias Sociales. Terminología Científico-Social, Tomo $1 / 2 / 3 / 4$, Ed. Plaza y Valdés, Madrid-México 2009. Disponible en: http://www. ucm.es/info/eurotheo/diccionario/M/metodocomparativo b. htm

VILLASANTE, Tomás. La socio-praxis: un acoplamiento de metodologías implicativas. Chile, 2004. Disponible en: http:// www.construyendocritica.uchile.cl/HTML/Textos/biblioteca/ La_socio-praxis, un acoplamiento_de_metodolog\%EDas implicativas.pdf $(20 / \overline{06} / 2012)$ 


\section{NOTES FROM A NEWCOMER FOR THINKING OF INVESTIGATION IN POLITICAL SCIENCE}

\author{
Corina Echavarría
}

We went from celebrating the participation of the people in case studies to needing to deepen the schemes for comprehension of the processes for broadening democracy which allow a compared analysis and the socialization of institutional and public learning. Thus, the part of the observing and analytical professional meets problems when developing actions of recognition and naming the world through science. In this work, we use the idea of the "newcomer" (M Fernandez) for questioning the situation of the foreignness of the investigator in comprehending and comparatively studying practices which are situated in a double dimension: the legitimacy of the knowledge which crosses the investigation, and the insertion of the otherness of the investigator in the processes of selfcomprehension of the collectives.

KEYwORDS: Public participation. Comparative method. Comprehension. Dialogue of knowledge. Activeresearch.

\section{NOTES D'UN “NOUVEAU-VENU” POUR PENSER L'INVESTIGATION EN SCIENCES POLITIQUES}

\section{Corina Echavarría}

Passer de la célébration de la participation citoyenne dans les études de cas au besoin d'approfondir les schémas de compréhension des processus d'amplification de la démocratie, permet de faire une analyse comparée et une socialisation des apprentissages citoyens et institutionnels. Dans ce cadre, le rôle du professionnel, qui observe et analyse, entre en conflit lorsqu'il réalise des actions de reconnaissance et doit désigner le monde à partir de la science. Dans cette étude, nous partons de l'image du "nouveau-venu" - M Fernandez pour remettre en question le fait que l'investigateur est considéré comme étranger à la compréhension et à l'étude comparée des pratiques situées dans une double dimension: la légitimité des savoirs qui se croisent dans ces processus de compréhension qui font partie de l'investigation et l'insertion de l'altérité du chercheur dans les processus d'autocompréhension des collectifs citoyens.

Mots-cLés: Participation citoyenne. Méthode comparative. Compréhension. Dialogues de savoirs. Recherche-action.

Corina Echavarría - Doutora em Administração. Professora da Faculdade de Ciência Política e Relações Internacionais da Universidade Católica de Córdoba e da Universidade Nacional de Córdoba. Pesquisadora do Conselho Nacional de Pesquisas Científicas e Técnicas (CONICET), Centro de Pesquisa e Estudos sobre Cultura e Sociedade (CIECS), da Universidade Nacional de Córdoba. Linha de pesquisa: problemas teóricos e empíricos associados a legitimidade deliberativo das instituições políticas e as práticas administrativas em sociedades complexas e pluralistas. 
